\title{
WAVE Approach for the Point Allocation on Examination
}

\author{
Hyeuk Kim \\ Assistant Professor, Department of Applied Statistics, Hoseo University, Asan, Korea
}

\begin{abstract}
There are two types of the items on examination. One test consists of the items with the same points and another test consists of the items with the different points. It is important to assign the points for the items based on a good standard when an examiner chooses an examination with the different points. The paper proposes the method which applies WAVE algorithm [1] in classification to point allocation on examination. WAVE algorithm is developed in combining the outputs of classifiers in ensemble. We apply it to the point allocation on examination since the concept of WAVE is similar with the concept of a tester's evaluation. It assigns high points on hard-to-solve items and low points on easy-to-solve items and considers an examinee's ability simultaneously.
\end{abstract}

Keywords: WAVE Algorithm, Point Allocation, Examination, Examinee's Evaluation

\section{INTRODUCTION}

An examination is the general method to evaluate examinees' abilities. It usually consists of the multiple items which have the corresponding points. An examinee gets the assigned point if he or she solves an item correctly and the evaluation is executed by the summation of the points which an examinee gets. It is important for an examiner to set a right standard in allocation of the points on examination. The valid evaluation of the examinees is impossible if the points in the items are allocated improperly. An examination is classified into two types based on the composition of the points of the items. The first type of an examination is that each item has the same point. The second type is that the items are assigned the different points.

A. Same points in the point allocation

It assigns the same points to all items on examination. The examinee's ability is judged by the summation of the number of the correct answers [2][3][4]. The approach is widely used since it is not easy to make an objective analysis for allocation of the different points.

\section{B. Different points in the point allocation}

It assigns the different points to the different items on examination. An examiner decides the standard for allocation of the points and assigns the different points to the different items based on the standard. One of the advantage in the approach is that it evaluates an examinee's performance in detail. Suppose that an examinee solves three items. There are four cases if the points in the items are same and each point is five. It can be $0,5,10$, or 15 . Suppose that points for the items are 3,5 , and 7 respectively. An examinee can get the eight possible total points which are $0,3,5,7,8,10,12$, or 15 . The number of the possible combination is twice as much asthe number of the possible combination in the same point allocation.
It is essential for an examiner to make a clear standard for the different point allocation on examination. There are several approaches which we review in the second section. The paper consists of five sections. We introduce the point allocation on examination in this section.

Three approaches for the different points allocation are described in the second section. WAVE algorithm is introduced and explained in the third chapter. We suggest the scenario how we apply WAVE algorithm in allocating points to the items on examination in the fourth section. We make a conclusion and discuss the future work in the final section.

\section{THREE APPROACHES IN THE DIFFERENT POINTS ALLOCATION}

We describe the approaches which allocates the different points to the items on examination. There are three approaches such as expert's decision, rational decision, and statistical decision [5].

\section{A. Expert's decision}

An expert assigns the points to the items based on his own knowledge. He is usually a specialist in the area on examination or the examination itself. It is possible that an examination results unexpectedly since an expert just makes items on examination and an examinee takes on exam actually. Moreover, it is hard to explain clearly why the items have the corresponding points in the approach since the standard for the point allocation is basically subjective.

\section{B. Rational decision}

It uses the objective standard for allocation of the points unlike expert's decision whose standard is determined subjectively. One of the most popular method in rational decision is to achieve the maximum of the reliability. 
Cronbach's alpha [6] is a measure for the reliability and it's formula is as follows:

$$
\alpha=\frac{\mathrm{K}}{\mathrm{K}-1}\left(1-\frac{\sum_{\mathrm{i}=1}^{\mathrm{K}} \sigma_{\mathrm{Y}_{\mathrm{i}}}^{2}}{\sigma_{\mathrm{X}}^{2}}\right)
$$

where $\sigma_{\mathrm{x}}^{2}$ is the variance of the observed total test scores, and $\sigma_{Y_{i}}^{2}$ is the variance of the $i$-th component for the sample of examinees. Kis the number of the items on examination.

\section{Statistical decision}

The method uses the advanced statistical analyses such as multiple regression analysis, discriminant analysis, and factor analysis. The objective of the approach is to find the appropriate points for the item to maximize the validity for examination.

\section{III.WAVE (WEIGHTED-ADJUSTED VOTING ALGORITHM FOR ENSEMBLES OF CLASSIFIERS)}

The allocation method using WAVE algorithm belongs to rational decision among three approaches. One of the rational decision approach is to assign the point based on the degree of the difficulty. A hard item is assigned a big point and an easy item is assigned a small point. However, WAVE algorithm consider an examinee's performance additionally to decide the points for the items. In an ordinary approach, the same points are given for two items if the numbers of the correct answers are same. The different points are assigned to two items when applying WAVE algorithm in the point allocation even though the numbers of the correct answers for two items are same.

WAVE is developed as the enhanced weighted majority voting algorithm in ensemble of classifiers. We consider the level of each item and the level of each examinee simultaneously and apply WAVE in classification to point allocation on exam in education. The following description is from Kim's doctoral dissertation [7].

\section{A. Iterative weight adjusting algorithm}

WAVE algorithm iterates the procedure to figure out the final weights by adjusting the weight for an item and the weight for an examineeby turns.

1. Set an initial item weight vector

$$
\mathrm{Q}_{0}=\frac{\left(\mathrm{J}_{\mathrm{nk}}-\mathrm{X}\right)\left(\mathrm{J}_{\mathrm{kk}}-\mathrm{I}_{\mathrm{k}}\right) 1_{\mathrm{k}}}{1_{\mathrm{n}}^{\prime}\left(\mathrm{J}_{\mathrm{nk}}-\mathrm{X}\right)\left(\mathrm{J}_{\mathrm{kk}}-\mathrm{I}_{\mathrm{k}}\right) 1_{\mathrm{k}}}
$$

$\mathrm{Q}_{0}$ takes higher item weights for $\mathrm{X}$ with fewer 1's (i.e., hard-to-classify items). The denominator is a normalizing factor for unit norm. It makes the summation of all weights in the vector to be 1 .

2. For $m=1,2, \ldots$, repeat (a) and (b).

(a) Calculate an examinee weight vector

$$
P_{m}=\frac{X^{\prime} Q_{m-1}}{1_{k}^{\prime} X^{\prime} Q_{m-1}}
$$

$\mathrm{P}_{\mathrm{m}}$ assigns higher weights on examinees who answer the correct answers more (i.e., columns of $\mathrm{X}$ with more 1's) after the item weight $Q_{m-1}$ is incorporated. The denominator is a normalizing factor for unit norm.

(b) Update the item weight vector.

$$
Q_{m}=\frac{\left(J_{n k}-X\right)\left(J_{k k}-I_{k}\right) P_{m}}{1_{n}^{\prime}\left(J_{n k}-X\right)\left(J_{k k}-I_{k}\right) P_{m}}
$$

$\mathrm{Q}_{\mathrm{m}}$ assigns higher weights on hard-to-answer item after incorporating the weight vector $P_{m}$ and $Q_{m}$ become stable. Denote $\mathrm{P}^{*}$ and $\mathrm{Q}^{*}$ as the final weight vectors. We already proved that these forms of the weight vectors always converged and their final weight vectors are as follows.

$$
\begin{gathered}
P^{*}=\lim _{m \rightarrow \infty} P_{m}=\lim _{m \rightarrow \infty} \frac{X^{\prime} Q_{m-1}}{1_{k}^{\prime} X^{\prime} Q_{m-1}} \\
=\frac{\left(\sum_{i=1}^{r} u_{i} u_{i}^{\prime}\right) 1_{k}}{1_{k}^{\prime}\left(\sum_{i=1}^{r} u_{i} u_{i}^{\prime}\right) 1_{k}}
\end{gathered}
$$

and

$$
\begin{array}{r}
Q^{*}=\lim _{m \rightarrow \infty} Q_{m}=\lim _{m \rightarrow \infty} \frac{\left(J_{n k}-X\right)\left(J_{k k}-I_{k}\right) P_{m}}{1_{n}^{\prime}\left(J_{n k}-X\right)\left(J_{k k}-I_{k}\right) P_{m}} \\
=\lim _{m \rightarrow \infty} \frac{\left(J_{n k}-X\right)\left(J_{k k}-I_{k}\right)\left(\sum_{i=1}^{r} u_{i} u_{i}^{\prime}\right) 1_{k}}{1_{n}^{\prime}\left(J_{n k}-X\right)\left(J_{k k}-I_{k}\right)\left(\sum_{i=1}^{r} u_{i} u_{i}^{\prime}\right) 1_{k}}
\end{array}
$$

where $r$ is the number of dominating eigenvalues of $\mathrm{X}^{\prime}\left(\mathrm{J}_{\mathrm{nk}}-\mathrm{X}\right)\left(\mathrm{J}_{\mathrm{kk}}-\mathrm{I}_{\mathrm{k}}\right)$ such that $\lambda_{1}=\lambda_{2}=\cdots=\lambda_{\mathrm{r}}>$ $\lambda_{\mathrm{r}+1}, 1 \leq \mathrm{r} \leq \mathrm{k}$ and $\mathrm{u}_{\mathrm{i}}$ is the eigenvector corresponding to the eigenvalue $\lambda_{i}, i=1, \ldots, r$.

\section{B. Toy Example}

If a tester solves the item correctly, then it is denoted by 1 in a performance matrix. Otherwise, it is denoted by 0 . Suppose that there are three testers and four items and a performance matrix $\mathrm{X}$ is given as

\begin{tabular}{|l|l|l|}
\hline 1 & 0 & 1 \\
\hline 1 & 1 & 1 \\
\hline 1 & 1 & 0 \\
\hline 0 & 0 & 1 \\
\hline
\end{tabular}

The eigen values for $\mathrm{X}^{\prime}\left(\mathrm{J}_{\mathrm{nk}}-\mathrm{X}\right)\left(\mathrm{J}_{\mathrm{kk}}-\mathrm{I}_{\mathrm{k}}\right)$ are 4.214, 1.461 , and 0.325 and the dominant eigen values is 4.214 . The eigenvector for the dominant eigenvector $u$ is $(-0.460$, $-0.143,-0.876)$. Therefore, we obtain

$$
\begin{gathered}
\mathrm{P}^{*}=\frac{\left(\sum_{\mathrm{i}=1}^{\mathrm{r}} \mathrm{u}_{\mathrm{i}} \mathrm{u}_{\mathrm{i}}^{\prime}\right) 1_{\mathrm{k}}}{1_{\mathrm{k}}^{\prime}\left(\sum_{\mathrm{i}=1}^{\mathrm{r}} \mathrm{u}_{\mathrm{i}} \mathrm{u}_{\mathrm{i}}^{\prime}\right) 1_{\mathrm{k}}}=(0.311,0.097,0.592)^{\prime} \\
\mathrm{Q}^{*}=\frac{\left(\mathrm{J}_{\mathrm{nk}}-\mathrm{X}\right)\left(\mathrm{J}_{\mathrm{kk}}-\mathrm{I}_{\mathrm{k}}\right)\left(\sum_{\mathrm{i}=1}^{\mathrm{r}} \mathrm{u}_{\mathrm{i}} \mathrm{u}_{\mathrm{i}}^{\prime}\right) 1_{\mathrm{k}}}{1_{\mathrm{k}}^{\prime}\left(\mathrm{J}_{\mathrm{nk}}-\mathrm{X}\right)\left(\mathrm{J}_{\mathrm{kk}}-\mathrm{I}_{\mathrm{k}}\right)\left(\sum_{\mathrm{i}=1}^{\mathrm{r}} \mathrm{u}_{\mathrm{i}} \mathrm{u}_{\mathrm{i}}^{\prime}\right) 1_{\mathrm{k}}} \\
=(0.311,0.000,0.140,0.548)^{\prime}
\end{gathered}
$$


The degrees of difficulty for four items are $0.311,0.000$, 0.140 , and 0.548 respectively. In an ordinary allocation of point, the first and the third items get the same points since two people out of three testers give the right answers for each item. However, we consider who answers correctly in WAVE algorithm. Therefore, the first item is assigned the higher point than the point of the third item since the first item is answered correctly by the first and the third testers who give three correct answers out of four items respectively. Meanwhile the first item is answered correctly by the first and the second testers. The first tester also solves both the first and the third item correctly. However, the second tester just gives two correct answers out of four items compared to the third tester who answers three items correctly.

It assigns zero point for the second item since all testers answer correctly. One of the objective on examination is to evaluate the examinees' performances. An item is useless for testing if all examinees give a right answer.

\section{IV.APPLICATION}

We describe how to apply WAVE algorithm in the point allocation on examination.

1. Create items for an examination:

An expert makes items to evaluate the examinees' performances.

2. Select the sample testers to decide the points for each item:

Any approaches except experts' decision need the sample testers to assign the points to the corresponding items. They have to represent examinees' characteristics well and the size of the sample has to be appropriate.

3. Apply WAVE algorithm:

We constitute a performance matrix from the sample testers' results which is introduced in the third section and apply WAVE algorithm

4. Determine the points for the corresponding items:

We calculate $Q^{*}$ in the proposed algorithm and figure out the points for each item based the weight vector $Q^{*}$.

\section{CONCLUSION AND FUTURE WORK}

There are several approaches how to assign the different points to the items on examination. The right standard for the point allocation is important for the evaluation unless an examination is useless. We propose to apply WAVE algorithm which is developed for combining the outputs of the classifiers in ensemble to the point allocation. We consider the level of each item and the level of each examinee simultaneously in WAVE algorithm. For future work we will make an experiment with real data and compute the reliability and validity. We will compare the performance of the proposed method with the performances of the existing methods such as multiple regression analysis, discriminant analysis, and factor analysis.

\section{REFERENCES}

[1] H. Kim, h. Kim, h. Moon, and h. Ahn, "a weight-adjusted voting algorithm for ensembles of classfiers,"journal of the korean statistical society, vol. 40, pp. 437-449, 2011.

[2] R. L. Ebel and d. A. Frisbie, essential of educational measurement, 5th ed., prentice-hall, 1991.

[3] J. P. Guilford, psychometric methods, 2nd ed., mcgraw-hill, 1954.

[4] H. Gulliksen, theory of mental tests, 1st ed., john wiley, 1950.

[5] M. W. Wang and j. C. Stanley, "differential weighting: a review of methods and empirical studies,"review of educational research, vol. 4, pp. 663-705, 1970.

[6] L. J. Cronbach, "coefficient alpha and the internal structure of tests," psychometrika, vol. 16(3), pp. 297-334, 1951.

[7] H. Kim, "a novel combining algorithm in classification," doctoral dissertation, 2013. 